\title{
R-process nucleosynthesis calculations with complete nuclear physics input
}

\author{
I. Petermann*; A. Arcones, A. Kelić, K. Langanke, G. Martínez-Pinedo, K.-H. Schmidt \\ GSI Helmholtzzentrum für Schwerionenforschung, Darmstadt, Germany \\ Institut für Kernphysik, TU Darmstadt, Germany
}

W. R. Hix

Physics Division, Oak Ridge National Laboratory, Oak Ridge, TN37831-6374, USA

I. Panov, T. Rauscher, F.-K. Thielemann

Department für Physik und Astronomie, Universität Basel, Switzerland

N. Zinner

Department of Physics, Harvard University, Cambridge, MA 02138

\begin{abstract}
The r-process constitutes one of the major challenges in nuclear astrophysics. Its astrophysical site has not yet been identified but there is observational evidence suggesting that at least two possible sites should contribute to the solar system abundance of r-process elements and that the r-process responsible for the production of elements heavier than $Z=56$ operates quite robustly producing always the same relative abundances. From the nuclear-physics point of view the r-process requires the knowledge of a large number of reaction rates involving exotic nuclei. These include neutron capture rates, beta-decays and fission rates, the latter for the heavier nuclei produced in the r-process. We have developed for the first time a complete database of reaction rates that in addition to neutron-capture rates and beta-decay half-lives includes all possible reactions that can induce fission (neutron-capture, beta-decay and spontaneous fission) and the corresponding fission yields. In addition, we have implemented these reaction rates in a fully implicit reaction network. We have performed r-process calculations for the neutrino-driven wind scenario to explore whether or not fission can contribute to provide a robust r-process pattern.
\end{abstract}

10th Symposium on Nuclei in the Cosmos

July 27 - August 12008

Mackinac Island, Michigan, USA

\footnotetext{
* Speaker.

$\dagger$ I. Petermann is supported by the Deutsche Forschungsgemeinschaft through contract SFB 634.
} 


\section{Introduction}

The r-process is a series of rapid neutron-capture reactions and beta-decays in explosive scenarios with high neutron densities. It is responsible for the synthesis of at least half of the elements heavier than Fe. Its astrophysical site has not yet been identified, but there is observational evidence suggesting the contribution of at least two sites, namely core collapse supernovae from stars of different masses and neutron-star mergers. Observed abundances of r-process elements with $Z>56$ in metal-poor stars follow a very robust pattern, that is consistent with scaled solar abundances[1]. Our aim is to explore whether or not fission cycling can provide an explanation for the robustness.

\section{Fission in the r-process}

In addition to neutron captures and beta decays a full set of fission rates [2], supplemented by the corresponding fission yields, are necessary. We have considered neutron-induced fission, computed using the SMOKER code [3], beta-delayed fission and spontaneous fission. For all of them fission yields have been computed using the statistical code ABLA [4], taking the neutrons evaporated explicitly into account. The resulting rates have been implemented in a fully implicit network, that includes approximately 7000 nuclei from $n, p$ up to ${ }^{300} \mathrm{Ds}$. The set of differential equations is linearized and solved using the Newton-Raphson method [5]. The inclusion of fission maintains the sparseness of the matrix and allows to use sparse matrix solvers, in our case the Parallel Sparse Direct Linear Solver (PARDISO) was used [6].

Fig. 1 shows the region where fission takes place during the r-process. Once the matter-flow breaks out of the magic neutron number $N=184$ the fission barriers decrease drastically, and fission becomes possible.
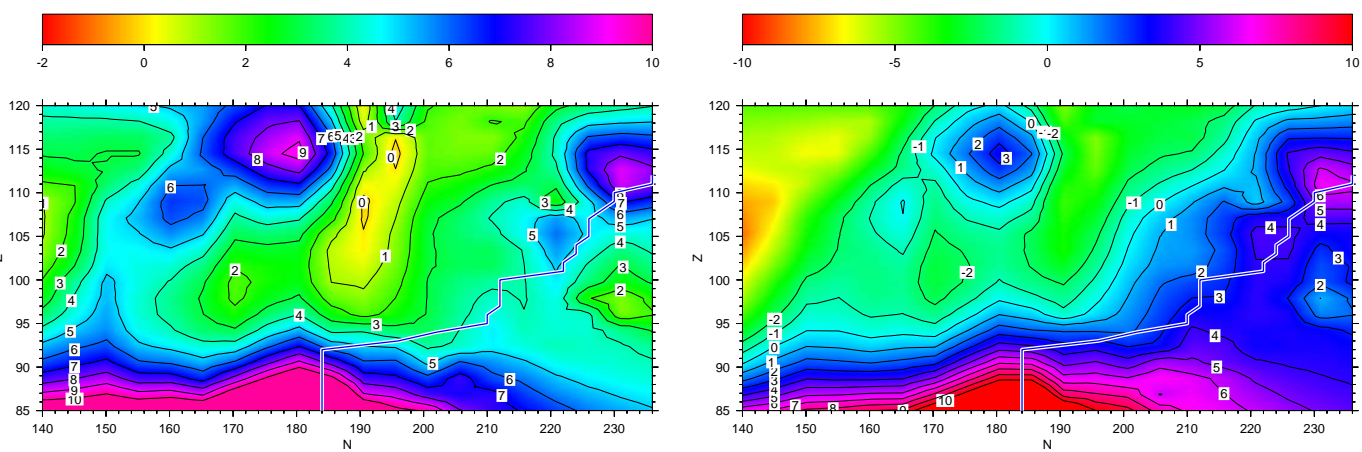

Figure 1: Region of the nuclear chart where fission takes place during the r-process. In the left panel the contour lines show the Myers-Swiatecki [7] fission barrier heights $\left(B_{f}\right)$ in MeV. The right panel shows contour lines of the quantity $B_{f}-S_{n}$, with $S_{n}$ the neutron separation energy taken from the FRDM mass model. The unlabeled line shows the location of the neutron drip-line using the FRDM mass model.

\section{Network calculations}

We have performed network calculations using trajectories obtained from model M15-11-r1 from the hydrodynamical simulations of ref. [8]. In order to achieve large enough neutron-to-seed 
ratios for a successful r-process we have increased the entropy obtained in these simulations. Our aim is to gain further insight in the influence that the inclusion of fission has in the observed final abundances. First, we try to determine what is the neutron-to-seed ratio necessary such as fission results in a substantial influence in the final abundances. As an estimate of the amount of fission that has occured we plot, in figure 2 , the number of fission cycles, defined as $n_{\text {cycl }}=\log _{2}\left(Y_{\text {final }} / Y_{\text {initial }}\right)$, where $Y_{\text {initial }}$ is the total abundance of nuclei with $A \geq 5$ after alpha-rich freeze-out and $Y_{\text {final }}$ is the abundance at the end of the calculation. At relatively low values of an initial neutron-to-seed ratio ( $\sim 150$ ), fission already accounts for $40 \%$ of the final abundance. This number depends on the mass model used. In all the calculations shown in this manuscript the FRDM masses [9] were used.

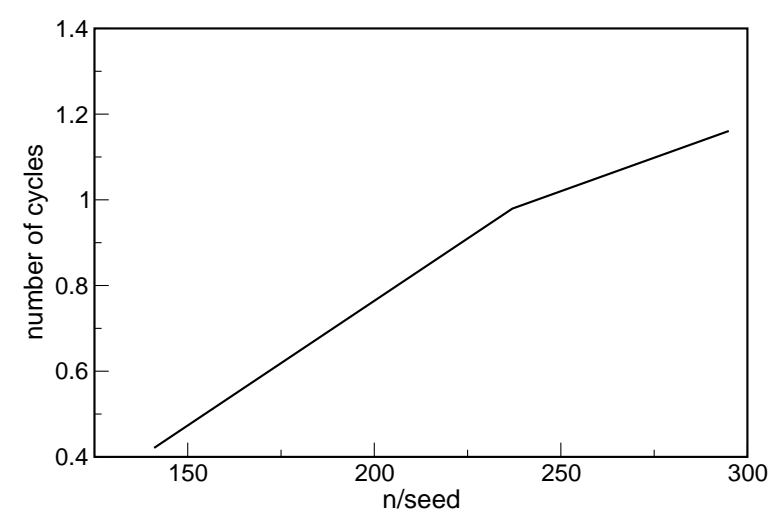

Figure 2: Number of fission cycles, for its definition see text, as a function of the neutron-to-seed ratio.

In addition to the total fission rate it is also interesting to determine what is the dominating fission channel, i.e. neutron induced fission, beta delayed fission or spontaneous fission. Fig. 3 shows the evolution of the fission rates for the different channels. They are computed determining the instantaneous increase in the total abundance of r-process nuclei, having $A \geq 5$, due to each fission channel. Clearly neutron induced fission is the dominating channel with beta delayed fission having a slight influence. The end of the r-process can be seen around $1.3 \mathrm{~s}$ by the sudden drop in the neutron-to-seed ratio.

The end of the r-process occurs at around $1.3 \mathrm{~s}$ and it is characterized by a sudden drop in the neutron-to-seed ratio. It is followed by a phase where all heavy fissioning nuclei decay over a timescale of several hundreds of seconds. During this phase neutron induced fission still dominates due to the neutrons produced by fission. Fig. 4 shows the elemental and isotopic abundances for nuclei with $A>190$ for three calculations with different initial neutron-to-seed ratios, $n /$ seed $=$ 140,237 and 295, at the time when the maximum mean value of $A$ is reached in each calculation. The heaviest nuclei produced have $Z \sim 94$ and $A \sim 280$ with $N=184$. According to our yield calculations, the fission products of these nuclei are two fragments with $A \sim 132,141$ and seven neutrons per fission event (see the dashed line in Fig.5).

The r-process abundances obtained after a time of around 30,000 yr are shown in Fig. 5 for different values of the neutron-to-seed ratio. Once the number of fission cycles becomes larger than one a robust r-process abundance distribution is obtained in the region below the $A \sim 195$ peak. The abundances in this region are mainly determined by the fission yield distribution. This distribution is practically the same for the different calculations as fission occurs in the same region 


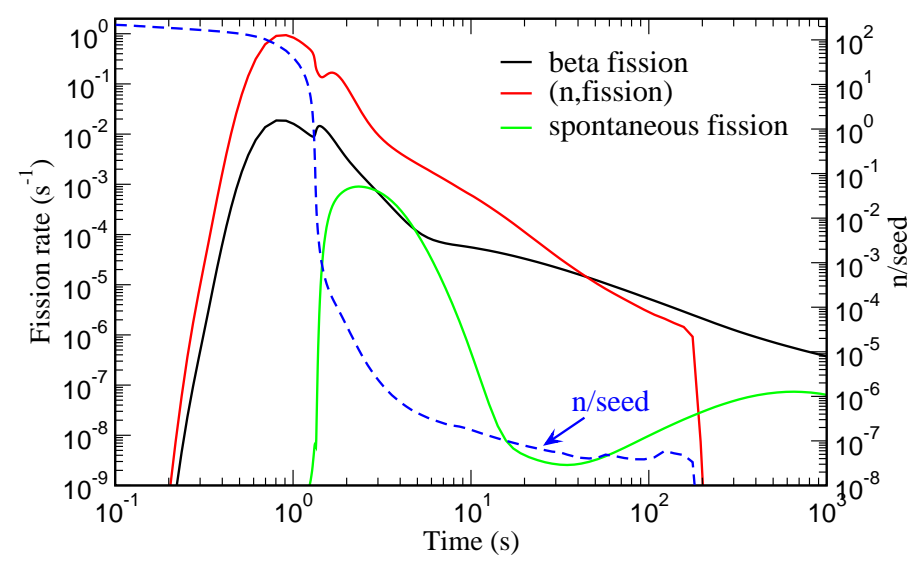

Figure 3: Evolution of the fission rate for the different channels for a calculation with initial neutron-to-seed ratio 295. Time zero corresponds to the beginning of the r-process phase after alpha-rich freeze-out. The evolution of the neutron-to-seed ratio is also shown (dashed line, right y-axis scale).
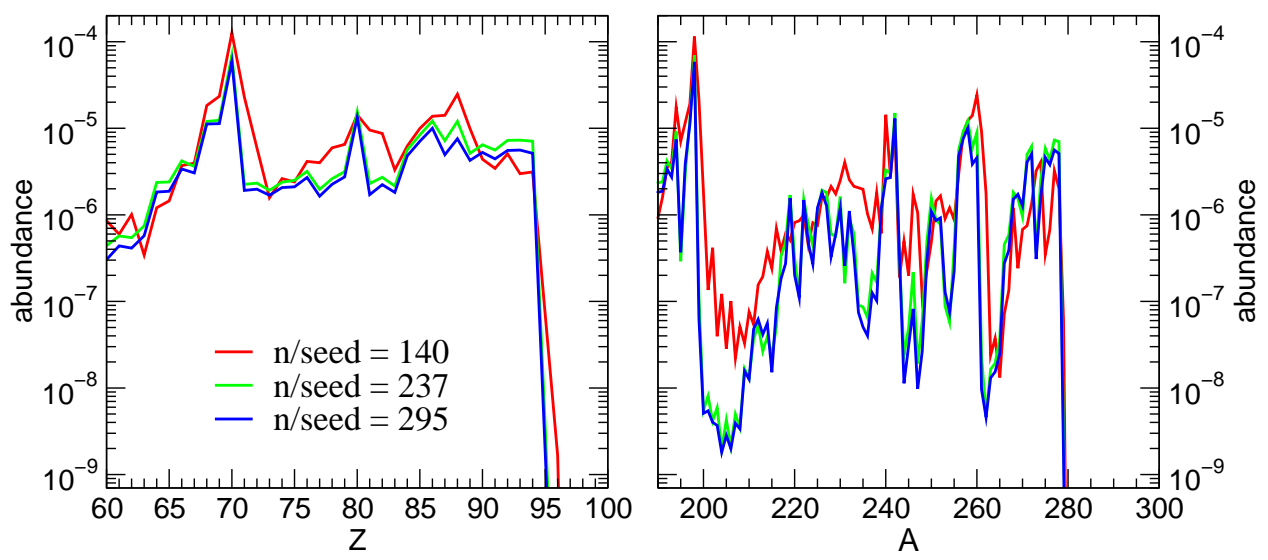

Figure 4: Elemental and isotopic abundance distributions for $Y_{\mathrm{n}} / Y_{\text {seed }}=140,237$ and 295.

of the nuclear chart (see fig. 4).

The dashed line shows the integrated contribution of fission to the isobaric abundances, defined as:

$$
Y(A)=\int d t \sum_{i} \lambda_{i}^{\mathrm{fis}}(A) Y_{i}
$$

where the sum runs over all the nuclei that can produce a nucleus with mass $A$ after fissioning with a rate $\lambda_{i}^{\text {fis }}(A)$. This distribution shows two peaks. The one at $A \sim 140$ is produced at early times when nuclei with $A \sim 280$ fission. At these times neutrons are still present and subsequent neutron captures distribute the fission yields over higher $A$-values. The peak at $A \sim 130$ is produced at later times when the neutron abundance is rather small and consequently is much less modified by neutron captures. 


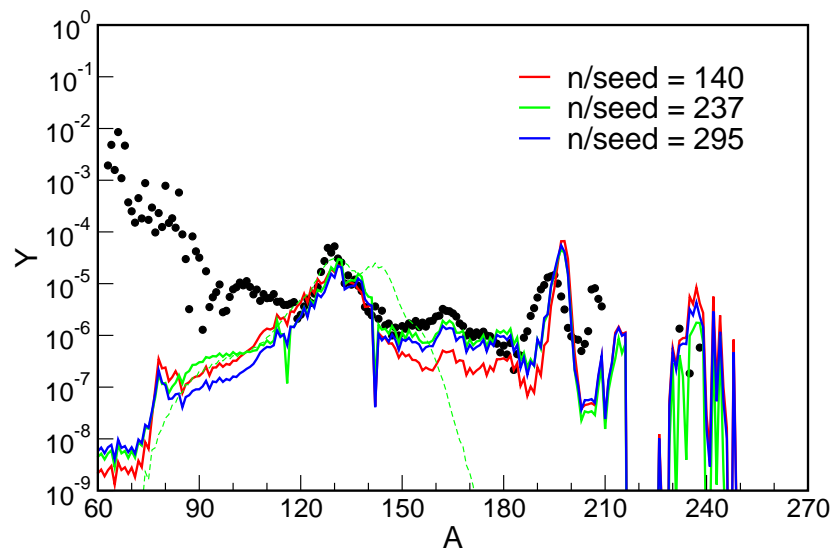

Figure 5: R-process abundances resulting from three calculations with different neutron-to-seed ratio (solid lines). The symbols show the r-process solar distribution. The dashed line shows the integrated fission yield distribution, see text for definition, obtained in the calculation with $\mathrm{n} / \mathrm{seed}=295$. The other calculations give almost identical distributions.

\section{Summary}

Using a full set of reaction rates that include all possible fission channels, supplemented with the corresponding fission yield distribution, we have explored the influence of fission in the $\mathrm{r}$ process. Our calculations show that neutron induced fission is the dominating channel. Similar results were obtained in ref. [10] in studies of r-process in neutron star mergers. The importance of fission has been quantified computing the number of fission cycles for each r-process calculation. This shows that already at relatively low initial neutron-to-seed ratios a noticeable part of the final abundance is due to fission events. Moreover, calculations with different neutron-to-seed ratios give very similar final abundance distributions for the mass model used in this work.

\section{References}

[1] J. J. Cowan and C. Sneden, Nature 440, 1151 (2006).

[2] J. J. Cowan, F.-K. Thielemann, J. W. Truran, Phys. Rep. 208 267-394 (1991).

[3] I. Panov et al., Nucl.Phys. A 747, 633-654 (2005). I. Panov, et al., in preparation.

[4] J.-J. Gaimard and K.-H. Schmidt, Nucl. Phys. A 531, 709 (1991).

[5] W. R. Hix, F.-K. Thielemann, Journal of Computational and Applied Mathematics 109, 321-351 (1999).

[6] O. Schenk and K. Gärtner, Journal of Future Generation Computer Systems 20, 475 (2004); O. Schenk and K. Gärtner, Elec. Trans. Numer. Anal. 23, 158 (2006); http: //www . pardiso-project.org.

[7] W. D. Myers and W. J. Swiatecki, Phys. Rev. C 60, 014606 (1999).

[8] A. Arcones, H.-Th. Janka, L. Scheck, A\&A, 467, 1227 (2007).

[9] P. Moller, J. R. Nix and W. J. Swiatecki, Atomic Data Nucl. Data Tables 59185 (1995).

[10] I. V. Panov and F.-K. Thielemann, Astronomy Letters 30, 647 (2004). 